${ }^{1}$ Clinic of Infectious and Tropical Diseases, University of Brescia, Brescia, Italy. ${ }^{2}$ The Global TB Programme, World Health Organization, Geneva, Switzerland. ${ }^{3}$ Dept of Public Health Sciences, Karolinska Institutet, Stockholm, Sweden. ${ }^{4}$ Migration Health Dept, International Organization of Migration, Geneva, Switzerland. ${ }^{5}$ WHO Collaborating Center for TB and Lung Diseases Fondazione S. Maugeri, Tradate, Italy. ${ }^{6}$ Public Health Consulting Group, Lugano, Switzerland.

Correspondence: Giovanni Battista Migliori, World Health Organization Collaborating Centre for Tuberculosis and Lung Diseases, Fondazione S. Maugeri, IRCCS, Via Roncaccio 16, 21049, Tradate, Italy.

E-mail: giovannibattista.migliori@fsm.it

Received: Sept 252015 | Accepted after revision: Sept 252015

Conflict of interest: Disclosures can be found alongside the online version of this article at erj.ersjournals.com

\title{
References
}

1 Lönnroth K, Migliori GB, Abubakar I, et al. Towards tuberculosis elimination: an action framework for low-incidence countries. Eur Respir J 2015; 45: 928-952.

2 International Organization for Migration (IOM). World Migration Report 2013. Geneva, International Organization for Migration, 2013.

3 Wild V, Zion D, Ashcroft R. Health of migrants: approaches from a public health ethics perspective. Public Health Ethics 2015; 8: 107-109.

4 Voniatis C, Migliori GB, Voniatis M, et al. Tuberculosis elimination: dream or reality? The case of Cyprus. Eur Respir J 2014; 44: 543-546.

5 Liu Y, Painter JA, Posey DL, et al. Estimating the impact of newly arrived foreign-born persons on tuberculosis in the United States. PLoS One 2012; 7: e32158.

6 Codecasa LR, Ciconali G, Mazzola E, et al. Managing an extensively drug-resistant tuberculosis outbreak: the public health face of the medal. Eur Respir J 2015; 45: 292-294.

$7 \quad$ Adapting to migration as a planetary force. Lancet 2015; 386: 1013.

8 UNHCR - The UN Refugee Agency. Refugees/Migrants Emergency Response - Mediterranean. http://data.unhcr. org/mediterranean/regional.php Date last accessed: September 18, 2015. Date last updated: August $31,2015$.

9 World Health Organization, Sixty-Seventh World Health Assembly. Draft global strategy and targets for tuberculosis prevention, care and control after 2015. A67/11. Geneva, World Health Organization, 2014.

10 D'Ambrosio L, Tadolini M, Centis R, et al. Supporting clinical management of the difficult-to-treat TB cases: the ERS-WHO TB Consilium. Int J Infect Dis 2015; 32: 156-160.

\section{Is there hope of improving the prognosis of pulmonary tumour thrombotic microangiopathy?}

To the Editor:

We read with interest the letter by KumAr et al. [1] reporting two new cases of fatal pulmonary tumour thrombotic microangiopathy (PTTM). As discussed by the authors, ante mortem diagnosis of PTTM is difficult to confirm because of a rapid progression of the disease. The disease is characterised histopathologically by microscopic tumour emboli and remodelling of the pulmonary vasculature, leading to right heart failure, severe hypoxaemia and, ultimately, death in the very short term, within a few hours or days following admission.

However, some cases have also been reported in the literature with higher survival of a few months that were not analysed in detail by Kumar et al. [1], who cited only one of these cases [2]. After reviewing all cases published in PubMed-indexed journals, since the original description by VON HerbAY et al. [3], we found six additional observations of such prolonged survival after a diagnosis of PTTM (table 1) [4-9]. These cases drew our attention because they could also be interesting to highlight the physiopathological mechanisms of PTTM and identify potential targeted therapies for this fatal condition.

One of these cases reported prolonged survival of a patient treated for a colorectal cancer with chemotherapy including bevacizumab, an anti-vascular endothelial growth factor (VEGF) monoclonal antibody [7]. VEGF is known to have a specific role in angiogenic endothelial cells and, thereby, in promoting the proliferation of endothelium involved in embryonic development and tumour angiogenesis. A recent clinical analysis of 30 autopsy cases observed that the immunohistochemistry of tumour cells 


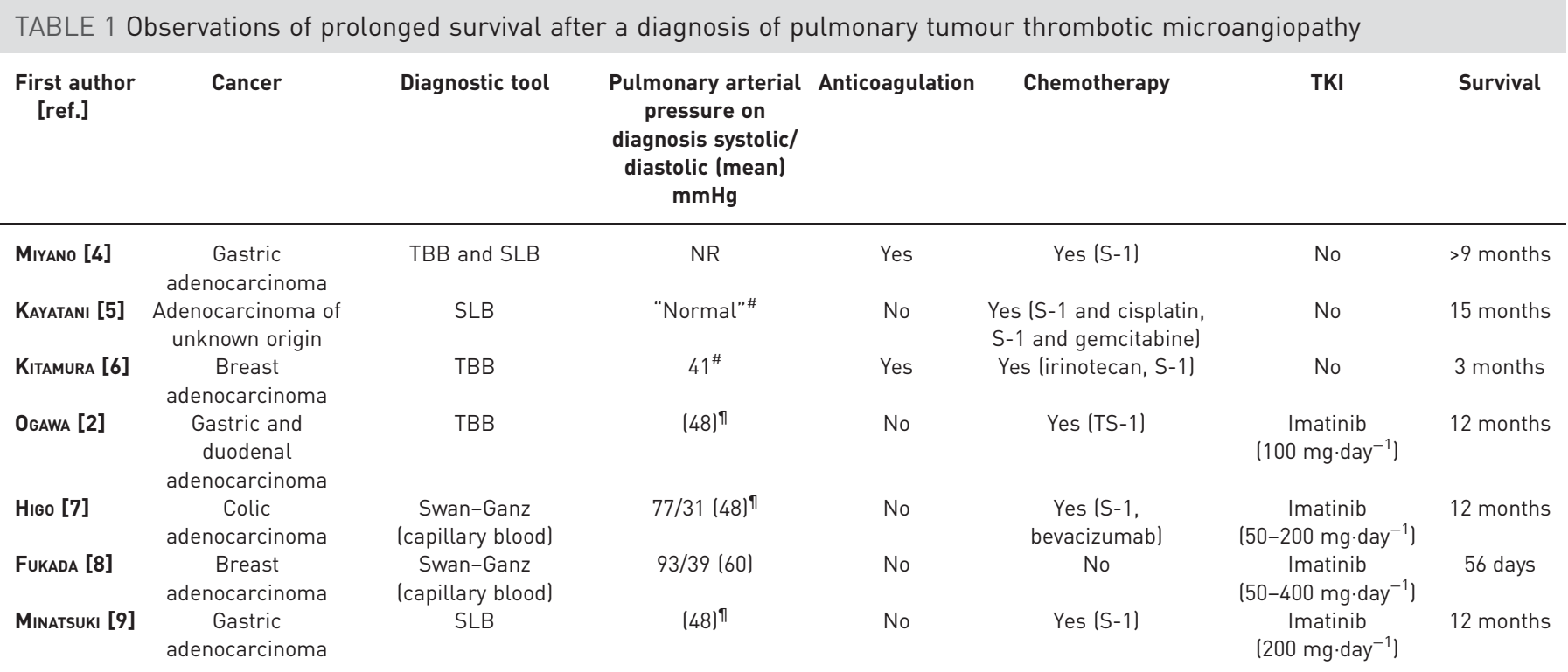

TKI: tyrosine kinase inhibitor; TBB: transbronchial biopsy; SLB: surgical lung biopsy; NR: not reported; S-1: fourth-generation fluoropyrimidine that contains tegafur, 5-chloro-2,4-dihidroxypyridine (gimeracil) and potassium oxonate (oteracil); TS-1: oral 5-fluorouracil derivative. \#: echocardiography (systolic); П : right hearth catheterisation.

located within the tumour emboli was positive for VEGF, suggesting that this pathway could be an interesting option to treat PTTM [10]. However, the effect of anti-VEGF antibody in this condition has to be evaluated in light of side-effects of this treatment, including thromboembolic events. In addition, four other patients were treated with imatinib, a tyrosine kinase inhibitor that inhibits platelet-derived growth factor (PDGF) receptors, at different dosages [2, 7-9]. Imatinib was associated in three cases with other chemotherapies acting on the VEGF pathway. In these patients, a survival of 12 months was reported, which is exceptional in PTTM. An immunohistochemical study reported PDGF and PDGF receptor expression in cancerous cells and pulmonary endothelial cells in lesions of PTTM caused by gastric carcinoma. In two cases, imatinib treatment lead to a decrease in serum PDGF concentration, which was associated with a decrease in D-dimer concentrations, despite the absence of anticoagulation [2,9]. Interestingly, in one case, a sustained clinical response after weaning from imatinib was observed, probably related to the efficacy of adjunct chemotherapy [2]. Again, the effect of this drug has to be evaluated in light of its side-effects. We have to remember that the risk/benefit ratio was recently considered unfavourable for the treatment of idiopathic pulmonary artery hypertension.

Based on these observations, we can hope that a better understanding of the pathophysiology of PTTM could result in specific targeted therapies that should improve prognosis of this vascular condition.

@ERSpublications

Better pathophysiological understanding of PTTM could result in specific targeted therapies and improve prognosis http://ow.ly/U93wg

Mansouria Merad ${ }^{1}$, Laurent Savale ${ }^{2}$, Sami Antoun ${ }^{1}$ and François Vincent ${ }^{3}$ for the GrrrOH: Groupe de Recherche en Réanimation Respiratoire en Onco-Hématologie (Group for Research in Respiratory Intensive Care Onco-Hematology)

${ }^{1}$ Service de Urgences et de Permanence des Soins, Gustave Roussy Cancer Campus Grand Paris, Villejuif, France.

${ }^{2}$ AP-HP, Service de Pneumologie, DHU Thorax Innovation, Hôpital Bicêtre, Le Kremlin-Bicêtre, France. ${ }^{3}$ Réanimation Polyvalente, GHIC Le Raincy-Montfermeil, Montfermeil, France.

Correspondence: François Vincent, Réanimation Polyvalente, GHIC Le Raincy-Montfermeil, 10, Avenue du Général Leclerc, 93370, Montfermeil, France. E-mail: frncsvncnt@aol.com

Received: Aug 152015 | Accepted after revision: Sept 242015

Conflict of interest: None declared.

\section{References}

1 Kumar N, Price LC, Montero MA, et al. Pulmonary tumour thrombotic microangiopathy: unclassifiable pulmonary hypertension? Eur Respir J 2015; 46: 1214-1217. 
2 Ogawa A, Yamadori I, Matsubara O, et al. Pulmonary tumor thrombotic microangiopathy with circulatory failure treated with imatinib. Intern Med 2013; 52: 1927-1930.

3 von Herbay A, Illes A, Waldherr R, et al. Pulmonary tumor thrombotic microangiopathy with pulmonary hypertension. Cancer 1990; 66: 587-592.

4 Miyano S, Izumi S, Takeda Y, et al. Pulmonary tumor thrombotic microangiopathy. J Clin Oncol 2007; 25: 597-599.

5 Kayatani H, Matsuo K, Ueda Y, et al. Pulmonary tumor thrombotic microangiopathy diagnosed antemortem and treated with combination chemotherapy. Intern Med 2012; 51: 2767-2770.

6 Kitamura A, Nishimura N, Jinta T, et al. A case of pulmonary tumor thrombotic microangiopathy diagnosed by transbronchial lung biopsy and treated with chemotherapy and long-term oxygen and anticoagulation therapies. Case Rep Pulmonol 2013; 2013: 259080.

7 Higo K, Kubota K, Takeda A, et al. Successful antemortem diagnosis and treatment of pulmonary tumor thrombotic microangiopathy. Intern Med 2014; 53: 2595-2599.

8 Fukada I, Araki K, Minatsuki S, et al. Imatinib alleviated pulmonary hypertension caused by pulmonary tumor thrombotic microangiopathy in a patient with metastatic breast cancer. Clin Breast Cancer 2015; 15: e167-e170.

9 Minatsuki S, Miura I, Yao A, et al. Platelet-derived growth factor receptor-tyrosine kinase inhibitor, imatinib, is effective for treating pulmonary hypertension induced by pulmonary tumor thrombotic microangiopathy. Int Heart J 2015; 56: 245-248.

10 Uruga H, Fujii T, Kurosaki A, et al. Pulmonary tumor thrombotic microangiopathy: a clinical analysis of 30 autopsy cases. Intern Med 2013; 52: 1317-1323.

Eur Respir J 2016; 47: 688-690 | DOI: 10.1183/13993003.01276-2015 | Copyright @ERS 2016

\section{From the authors:}

We thank M. Merad and coworkers for their comments. Indeed there have been at least eight reported cases of pulmonary tumour thrombotic microangiopathy (PTTM) since ours was submitted in early 2015, including cases in other major respiratory journals [1], emphasising that PTTM may not be such a rare condition. Indeed, it is being increasingly recognised both by oncologists and pulmonary hypertension physicians alike. Of over two hundred case reports or series of PTTM now cited on PubMed, the majority relate to adenocarcinomas, mainly gastric in origin, but also oesophageal, colorectal, pancreatic or lung, and more rarely breast, bladder and ovarian. Autopsy studies suggest a prevalence of $3 \%$ in gastric cancer [2]. Despite increasing recognition, however, most cases remain a post mortem diagnosis. The presence of undiagnosed malignancy must therefore be borne in mind when physicians assess new cases of pulmonary hypertension.

Early diagnosis is clearly important. We agree with the authors' hypothesis that identification of pathways involved in the pathogenesis of PTTM may improve survival by allowing targeted therapy. In addition to the vascular endothelial growth factor and platelet-derived growth factor pathways there is evidence for dysregulated intravascular coagulation as indicated by activation of tissue factor and raised D-dimer. As well as increased awareness, we also need an improvement in diagnostic accuracy.

In a new patient presenting with pulmonary hypertension of unknown cause, undiagnosed malignancy should be in the differential diagnosis. The clinical features of PTTM include non-specific signs of pulmonary hypertension but may include symptoms and signs of malignancy in other organs. In terms of blood tests, the role of common tumour markers is not established in the routine work-up of a new patient with suspected pulmonary hypertension. Importantly, the presence of a raised D-dimer with an absence of filling defects seen on computed tomography pulmonary angiography [3] is described in PTTM. Ventilation-perfusion scanning, however, may demonstrate distal and non-segmental diffuse perfusion abnormalities [4]. Conventional pulmonary angiography may show occlusion of distal vessels but without abrupt narrowing or intravascular webs suggestive of chronic thromboembolic pulmonary hypertension [5]. High-resolution computed tomography findings are non-specific but may demonstrate ground glass opacification, mosaicism (reflecting small vessel occlusion) [5], small pulmonary nodules (due to fibrointimal thickening), diffuse shadows [6], consolidation, tree-in-bud [7] and interlobular septal thickening (when pulmonary vein or lymphatics are involved). Interestingly, the recent European Society of Cardiology/European Respiratory Society pulmonary hypertension guidelines suggest that computed tomography is useful in many respects, but does not emphasis the potential role for computed tomography (and/or ultrasound) of the abdomen and pelvis to assess for malignancy [8]. Indeed, as previously highlighted previously in this journal, computed tomography should pick up malignancy in rapidly progressive new cases of pulmonary hypertension [9]. The presence of signs of pulmonary veno-occlusive disease on high-resolution computed tomography (including interstitial oedema with diffuse central ground-glass opacification and thickening of interlobular septa) in a patient with likely 